\title{
Prolonged neurologic dysfunction after a general anaesthetic
}

\author{
Chandni Rajani, Su Cheen $\mathrm{Ng}^{*}$, Navkiran Kaur
}

University College London Hospital NHS Foundation Trust, London, United Kingdom

\begin{abstract}
We report a case of a twenty year old Caucasian male student with no past medical history, who underwent a general anaesthetic for a knee arthroscopy. Post-operatively, he developed a prominent and debilitating stutter. He was unable to formulate sentences, and could solely communicate via writing. Organic causes of this presentation were excluded following a full neurological assessment and an MRI scan of the brain. He was subsequently diagnosed with a functional speech disorder. The stutter prolonged his hospital stay by three days and persisted for four months. His speech eventually normalised following an extensive period of intensive speech therapy. To our knowledge, this is the first reported case of a functional speech disorder following general anaesthesia.
\end{abstract}

Keywords: speech disorder, general anesthesia, recovery, awareness

\section{Introduction}

To our knowledge, there are no reported cases of a stutter of this nature occurring after a general anaesthetic, in an otherwise previously well patient with no past medical or psychiatric history. This case is of particular interest due to the thorough and efficient diagnosis and management of a rare but debilitating presentation.

\section{Case report}

A twenty year-old Caucasian male university student presented for an uncomplicated forty minute day-surgery intervention for a knee arthroscopy. There was no past medical, drug or alcohol history. The patient underwent induction of anaesthesia with 100 microgram fentanyl, 1.5 milligram

Received: November 2016; Accepted after review: March 2017; Published: March 2017.

${ }^{*}$ Corresponding author: Su Cheen $\mathrm{Ng}$, University College London Hospital NHS Foundation Trust, London, UK

E-mail: sucheenng@gmail.com midazolam and 200 milligram propofol to facilitate spontaneous ventilation via a Classic Laryngeal Mask Airway. Anaesthesia was maintained using oxygen, air and sevoflurane. Paracetamol 1 gram, diclofenac 75 milligram, ondansetron 4 milligram and a further 100 microgram of fentanyl were administered intravenously intra-operatively. During the procedure the patient was stable recording a systolic blood pressure above $90 \mathrm{mmHg}$ throughout with minimal fluctuations. Minimum alveolar concentration for Sevoflurane was between 1.1 and 1.2 throughout the surgical procedure. No depth of anaesthesia monitoring was utilized.

On waking in the post anaesthetic care unit, the patient reported severe pain in his operated knee and felt he could not move his leg. He was unable to articulate full sentences, stuttered and was shaking profusely. He understood and processed questions directed to him but exhibited difficulty in verbalising, but not word finding, thus wrote down his responses. Clinical signs did not improve on optimisation of his analgesia. Twenty milligrams of morphine and a hundred micrograms of fentanyl were administered in 
the first two hours of recovery. Despite his pain subsiding and having full movement of his lower limbs, he was unable to talk without a prominent stutter. An urgent neurological consultation was sought: both neurological examination and subsequent MRI brain scan revealed no abnormality. On day three postsurgery he was discharged home with a diagnosis of functional speech disorder.

$\mathrm{He}$ received intensive community-based speech therapy sessions for four months, eventually leading to a full recovery. Progress was steady throughout and he was regularly followed up by his general practitioner, the orthopaedic and anaesthetic teams via outpatient appointments and follow-up phone calls.

The stutter persisted for four months but the patient eventually made a full recovery to return to his academic life.

This was an extremely distressing and anxious time for the patient and his family as it led to a three-day prolongation in his hospital stay. It had significant impact on his social life and academia preventing him from returning to university for four months.

The event also posed a significant challenge for the anaesthetic and orthopaedic teams, with regards to finding a potential cause for this presentation alongside formulation of an optimum management plan. On follow-up eight months later, the patient has settled back into his Physics degree and just returned from a two-month trip around Europe, with no residual speech disturbance.

\section{Discussion}

We report a functional speech disorder that manifested as stuttering following general anaesthesia (GA). To our knowledge, this has not been previously reported in the medical literature.

Stuttering is a form of speech disorder, characterized by marked disruption to the flow of speech. There are noticeable prolongations in pauses during speech, as well as repetitions of sounds, words and phrases. Stuttering can be developmental or acquired. Developmental stuttering is the most common form of stuttering and begins in childhood or adolescence. Two main types of acquired stuttering have been described: neurogenic and psychogenic. The latter is thought to be associated with a coinciding emotional stressor or a traumatic event, whereby a psychological stressor manifests as a somatic symptom. In contrast to developmental stuttering, which is more common in males (3:1 ratio), psychogenic or functional stuttering is thought to occur in males and females in an equal ratio [1].

Functional speech disorders can present in many forms ranging most often as stuttering, dysphonia and prosodic (rhythm, speed and relative emphasis) abnormalities. There are no reported stuttering phenomenons after GA however four cases of aphemia or mutism after GA have so far been published in the literature [1-4]. Narayanan reported a case of a forty year old, previously fit and well female becoming 'speechless' for one day after an uncomplicated general anaesthetic for an emergency Caesarean Section [2]. Differential diagnoses such as cerebral sinus venous thrombosis, reversible cerebral vasoconstriction syndromes and postpartum angiopathy were excluded. Of note, the presentation for these conditions was aphasia and not aphemia and no other organic brain lesions cause was found. Raphael and Schoenfield described the case of a forty four year old lady with a background of bipolar affective disorder, obesity and a history of childhood sexual abuse who presented with a four month history of stuttering two years after Roux-en-Y gastric bypass operation [1]. The authors concluded that patients undergoing this procedure with a history of psychiatric illness and childhood sexual abuse might be predisposed to 'somatoform disorders'. General anaesthesia, however, was not highlighted as a contributing or causative factor. The third case involved a fifty six year old female patient undergoing an umbilical hernia repair [3]. The patient already had one documented episode of inability to speak after a recent GA that was attributed to the use of ketamine. After emergence from GA for hernia repair, she presented with mutism alongside weakness of limbs although no organic cause was found. She subsequently made a full recovery. The fourth case is slightly different. 
A female patient underwent total intravenous anaesthesia for femur fracture fixation [4]. She developed mutism for eleven days postoperatively but then made a full recovery by day eighteen-post operation. In this case no neurologic abnormality was obtained other than minimal cerebral oedema that resolved on day five. The authors did not attribute the cerebral oedema to mutism. They concluded that propofol could have been the contributing factor to the speech disorder.

Other differential diagnosis that manifests as speech disorders should also be sought. Drug-induced causes and awareness under GA may lead to post traumatic stress disorder (PTSD) and should be considered in such circumstances.

Pharmacogenic or drug-induced stuttering has been reported with a multitude of medications. A review for drug-induced stutter carried out on the French pharmacovigilance database over a 29-year period reported that antiepileptic, neuroleptic and antidepressive drugs were the main agents implicated [5]. Cholinergic, dopaminergic, noradrenergic and serotonergic mechanisms have been implicated in the pathophysiology for druginduced stutter [6]. Interestingly medications used for treatment for stuttering could also to be implicated in the occurrence of stuttering [7, 8]. In the reported cases for medicationinduced stutter, it was noted that patients recovered from the speech impediment when the medication was ceased. In this presented case, our patient only made full speech recovery after 4 months of the first presentation. Whilst there are multiple case reports of drug-induced stuttering, the incidence of speech disorders after emergence from GA for non-neurological surgeries is rare.

Awareness can be either explicit memory (conscious recollection) or implicit memory (no conscious recollection). Approximately $0.5 \%$ to $2 \%$ of surgical patients undergoing GA may experience explicit recall [9]. A much more complex association however exists between implicit memory and patients undergoing GA. These patients are at a risk for developing anxiety symptoms that may be transient or can lead to PTSD. This can last for many years and cause significant discomfort or impairment in social functioning and daily life. In some cases, patients may require long-term medical and psychological treatment [9]. The recent NAP 5, which is the largest study on awareness so far, highlights cesarean sections and cardiothoracic subgroups as the higher risk groups. Awareness under GA in the study was found to be 1:8 000 when neuromuscular blockade was used and significantly lower at 1:136 000 when no paralysis was involved [10]. In our case report, our patient received no neuromuscular blockade for his surgery. Depth of anaesthesia monitoring was not used on our patient either.

The critical step in management of a functional speech disorder is institution of the appropriate medical care. Furthermore, the ability to differentiate functional speech disorders from speech disorders that stem from organic pathology is indispensable in the development of appropriate speech therapy structured programs. From a clinician's perspective, this is determined by swift detection of the problem followed by appropriate referral for therapy. Baumgartner and Duffy further investigated the differentiation between neurogenic and psychogenic stuttering. Their work outlined notable differences that would aid a clinician in distinguishing neurogenic from psychogenic stuttering. Dysfluencies (repetitions of sounds and syllables, prolongations), unpredictable variability in speech and situational variability were thought to be more prevalent in the psychogenic stuttering group without neurological organic involvement. Conversely, interjections in stuttering and time of day variability remained relatively constant between both groups. It was further noted that a prominent distinction between both types of stuttering is the rapidity in a patient's ability to respond to therapy. Their work highlighted that patients with psychogenic stuttering responded better and recovered far more quickly than those in the neurogenic category [11]. 


\section{Conclusions}

Early recognition of an abnormality in recovery and a thorough post-operative neurological assessment are important in enabling early exclusion of possible organic, neurologically-based causes.

Acquired stuttering could be due to many potential factors and a thorough assessment

\section{References}

1. Raphael DB, Schoenfeld FB. Psychogenic stuttering following a gastric bypass operation: case report. Jefferson J Psychiatry 2006; 20:13-21.

2. Narayanan A, Tawfic QA, Kausalya R, et al. Speechless after general anaesthesia for caesarian section. MEJ Anesth 2012; 5:739741.

3. Gurunathan $\mathrm{U}$, Iswariah $\mathrm{H}$. A case of mutism on emergence from general anesthesia. $J$ Anesth 2016; 30:545.

4. Kati I, Demirel CB, Anlar O, et al. An unusual complication of total intravenous anesthesia: mutism. Anesth Analg 2003; 96:168-170.

5. Béné J, Auffret $M$, Fedrizzi $S$, et al. Druginduced stuttering: a review of the French Pharmacovigilance Database. Drug Safety 2014; 37:875.

6. Brady JP. Drug-induced stuttering: a review of the literature. J Clin Psychopharmacol 1998; 18:50-54. including psychological testing should be considered.

The effectiveness of the ongoing multidisciplinary support provided to the patient both during his illness and after recovery is important. Such psychological support can aid in alleviating the frightening journey a patient faces during such adverse circumstance.

7. Nurnberg HG, Greenwald B. Stuttering: an unusual side effect of phenothiazines. $A m \mathrm{~J}$ Psychiatry 1981; 138:386-387.

8. Adler L, Leong S, Delgado R. Drug-induced stuttering treated with propranolol. J Clin Psychopharmacol 1987; 7:115-116.

9. Aceto P, Perrili V, Lai C, et al. Update on Post traumatic stress syndrome after anaesthesia. Eur Rev Med Pharmacol Sci 2013; 17:17301737.

10. Pandit J, Cook T. 5th National Audit Project (NAP5) on accidental awareness during general anaesthesia: summary of main findings and risk factors. $\mathrm{Br} J$ Anaesth 2014; 113(4):549-59.

11. Baumgartner J, Duffy JR. Psychogenic stuttering in adults with and without neurologic disease. J Med Speech Lang Pathol 1997; 5:75-95. 